\title{
A comparison between remaining and resecting the posterior longitudinal ligament in percutaneous endoscopic transforaminal discectomy for disc herniation: A retrospective cohort study
}

\section{Wenhao Hu}

Fourth Medical Center of PLA General Hospital

\section{Fanqi Hu}

Fourth Medical Center of PLA General Hospital

Chao Liu

Fourth Medical Center of PLA General Hospital

Weibo Liu

Fourth Medical Center of PLA General Hospital

\section{Yi Jiang}

PLAGH: Chinese PLA General Hospital

Jing Li

Fourth Medical Center of PLA General Hospital

\section{Yan Wang}

Fourth Medical Center of PLA General Hospital

\section{Teng Li}

Fourth Medical Center of PLA General Hospital

Li Li

Fourth Medical Center of PLA General Hospital

\section{Xuesong Zhang ( $\boldsymbol{\nabla}$ 578437377@qq.com )}

Chinese PLA General Hospital

\section{Research article}

Keywords: percutaneous endoscopic lumbar discectomy, percutaneous endoscopic transforaminal discectomy, the posterior longitudinal ligament, Visual analog scale, Oswestry disability index

Posted Date: September 9th, 2020

DOI: https://doi.org/10.21203/rs.3.rs-74154/v1 
License: (c) (i) This work is licensed under a Creative Commons Attribution 4.0 International License. Read Full License 


\section{Abstract \\ Background}

Nowadays, percutaneous endoscopic lumbar discectomy (PELD) has become a popular surgical option for the management of LDH, On the basis of the approach to the herniation disc materials, PELD could be classified into percutaneous endoscopic transforaminal discectomy (PETD) and percutaneous endoscopic interlaminar discectomy (PEID). During PETD procedure, especially in in-and-out approach, accurate determination of whether the posterior longitudinal ligament is needed resection seems particularly important.In the study, we tried assessing comparative clinical outcome, surgical duration and complications between the two different surgical methods of LDH treated by PETD via inside-and-out approach.

\section{Methods}

A total of 135 symptomatic LDH patients who were treated by PETD resecting the posterior longitudinal ligament (68 patients) or remaining the posterior longitudinal ligament (67 patients) during the surgery were included in the study. Visual analog scale (VAS) score, Oswestry disability index (ODI) and modified MacNab criteria were used to evaluate clinical outcomes. Operation time, blood loss, recurrence of LDH and other complication such as dural tear, nerve injury, intervertebral infection, etc. of these two groups were recorded. Clinical evaluation was performed preoperatively, immediately postoperatively, and at each follow-up visit (1 month, 3 months, and final follow-up).

\section{Results}

There were 38 males and 30 females in the group A that resecting the posterior longitudinal ligament (mean age $=52.40 \pm 8.73$ years) and 35 males and 32 females in the group $B$ that remaining the posterior longitudinal ligament (mean age $=53.50 \pm 9.24$ years). The mean operation time of the group $A$ and the group $B$ respectively, were $82.7 \pm 18.5 \mathrm{~min}$ and $115.6 \pm 24.6 \mathrm{~min}(P<0.01)$. The postoperative VAS scores and ODI were significantly higher than those before surgery in two groups $(P<0.01)$. The differences in the results were not significant between the two groups $(P>0.05)$. There was no significant difference in the complication rate, the excellent/good ratio and the recurrence rate between the two groups $(P>0.05)$.

\section{Conclusion}

Whether the posterior longitudinal ligament is resected do not make any difference on curative effect in PETD via inside-and-out approach, and remaining the ligament when it's intact is preferred because of its shorter operation time. 


\section{Background}

Lumbar disc herniation (LDH) is a common disease with a reported prevalence of $1-3 \%$ and is a major cause of low back pain (LBP) with radiation to the legs[1]. Although conservative treatment has been usually proposed for LDH patients, some patients whose symptoms has not improved with conservative management may still require surgical intervention[2]. Among the operative methods, open lumbar discectomy and fusion is considered to be the gold standard treatment of $\operatorname{LDH}[3,4]$.However, the procedure make damage to muscular and ligamentous structures and require prolonged hospital stays and expensive hospitalization cost. Nowadays, percutaneous endoscopic lumbar discectomy (PELD) has become a popular surgical option for the

management of LDH, Since the posterolateral nucleotomy technique was first introduced by Kambin and Gellman[5-7] .

On the basis of the approach to the herniation disc materials, PELD could be classified into percutaneous endoscopic transforaminal discectomy (PETD) and percutaneous endoscopic interlaminar discectomy (PEID). PETD is more widely used because it can manage all types of LDH theoretically[8]. During PETD procedure, especially in in-and-out approach, accurate determination of whether the posterior longitudinal ligament is needed resection seems particularly important. To our knowledge, there is not much research to cormpare the curative effect of resecting the posterior longitudinal ligament or not.

In this retrospective study, we tried assessing comparative clinical outcome, surgical duration and complications between the two different surgical methods of LDH treated by PETD via inside-and-out approach.

\section{Materials And Methods}

From January 2015 to January 2017, a total of 135 symptomatic LDH patients who were treated by PETD resecting the posterior longitudinal ligament (68 patients) or remaining the posterior longitudinal ligament (67 patients) during the surgery were included in this retrospective analysis. This study was conducted with approval from the Ethics Committee of our Hospital. Written informed consent was obtained from all participants. All procedures involving human participants were performed in accordance with the Declaration of Helsinki. Visual analog scale (VAS) score, Oswestry disability index (ODI) and modified MacNab criteria were used to evaluate clinical outcomes. All cases underwent lumbar spine anteroposterior, lateral, flexion-extension x-ray, computed tomography (CT), and magnetic resonance imaging (MRI) to confirm the diagnosis and pathological type. Operation time, blood loss, recurrence of LDH and other complication such as dural tear, nerve injury, intervertebral infection, etc. of these two groups were recorded. Clinical evaluation was performed preoperatively, immediately postoperatively, and at each follow-up visit (1 month, 3 months, and final follow-up). 3 months after surgery, MRI was performed to confirm the nerve root compression was relieved and flexion-extension $\mathrm{x}$ ray was used to assess spinal stability. All patients were followed up without loss for at least 24 months. 
The inclusion criteria were as follows: (1) symptomatic radiating leg pain with positive straight leg-raising test; (2) CT and MRI suggesting a single level disc herniation correlated with the clinical findings; (3) significant pain refractory to conservative treatment for at least 4 weeks such as bed rest, immobilization, and pain management program; (4) no previous surgical history at the same level. The exclusion criteria were as follows: (1) flank pain as the main symptom; (2) segmental lumbar spinal stenosis or degenerative scoliosis; (3) unstable lumbar vertebrae; (4) rupture of the posterior longitudinal ligament preoperatively (5) high iliac crest or large L5 transverse process which interlaminar approach required; (6) infection, tumor or other pathological conditions.

\section{Operative Technique}

All surgeries were performed by the same experienced surgeon. Patients were placed in the prone position on a radiolucent surgery table. Local anaesthesia was selected for monitoring of any intra-operative changes in the patients'symptoms and signs. Prior to surgery, C-arm X-ray fluoroscopy was used to confirm the target segment. The surgical puncture point and distance depended on the body type of each patient, and the distance was usually $11-14 \mathrm{~cm}$ from the midline. $1 \%$ Lidocaine was used from the skin fascia to the surface of the annulus fibrosus. An 18G needle was inserted into target intervertebral foramen. Anteroposterior fluoroscopy confirmed the needle positioned on the medial pedicle edge. Lateral fluoroscopy confirmed the needle positioned on the posterior edge of the vertebral body. Then, Endoscope was positioned through a working channel that was inserted via a $0.8-\mathrm{cm}$ skin incision at the entry point of the puncture needle (Fig. 1).

Under endoscopy, the posterior longitudinal ligament was usually in the middle of the field, and the spinal canal is up, the intervertebral disc is down(Fig. 2). First, pituitary rongeur was used to remove the prominent intervertebral disc under the posterior longitudinal ligament. Next, the working channel was rotated upward to detect residual nucleus pulposus tissue in spinal canal. During the procedure, the posterior longitudinal ligament was resected for some patients (68 cases). Decompression is supposed to sufficient when the nerve root has good mobility and the dural sac beat with the heartbeat. Bipolar radiofrequency was used for Annulus fissure coagulation and hemostasis. After endoscopy and working channel were removed, the skin was sutured with a single stitch.

All PETD is daytime surgery at our hospital, and patients were discharged after resting in bed for 1-2 hours postoperatively. 1 day later, patients were recommended to begin lumbodorsal muscle exercise and straight leg-raising exercise with a protective belt.

\section{Statistical Analysis}

Statistical analyses were carried out using SPSS version 19.0 software (SPSS, Chicago, IL). The continuous variables were expressed as mean \pm standard deviation. The intergroup operation time, blood loss sample means were compared with $t$ test. The intragroup preoperative and postoperative VAS and ODI were compared with paired t test and the Wilcoxon rank sum test. Intergroup satisfaction scores, 
recurrence of LDH and complications used the $x^{2}$ test. The result was considered statistically significant if $P<0.05$.

\section{Results}

PETD via inside-and-out approach were performed in all patients. 135 patients were grouped to undergo either of the surgical methods. There were 38 males and 30 females in the group $A$ that resecting the posterior longitudinal ligament (mean age $=52.40 \pm 8.73$ years) and 35 males and 32 females in the group $B$ that remaining the posterior longitudinal ligament (mean age $=53.50 \pm 9.24$ years). There were no statistically significant differences between the two groups in demographic data such as age, gender, or pain duration (Table 1).

Table 1

पGeneral characteristics of the patients

\begin{tabular}{|llll|}
\hline Item & Group A & Group B & P value \\
\hline Gender M/F & $38 / 30$ & $35 / 32$ & $>0.05$ \\
\hline Age (yrs) & $52.40 \pm 8.73$ & $53.50 \pm 9.24$ & $>0.05$ \\
\hline Surgical Segment ( $\mathrm{n}, \%)$ & & & $>0.05$ \\
\hline L3/4 & $6(8.8 \%)$ & $7(10.4 \%)$ & \\
\hline L4/5 & $51(75 \%)$ & $49(73.1 \%)$ & \\
\hline L5/S1 & $11(16.2 \%)$ & $11(16.4 \%)$ & \\
\hline Types(n, \%) & & & \\
\hline central & $7(10.3 \%)$ & $5(7.5 \%)$ & \\
\hline paracentral & $32(47.1 \%)$ & $30(44.8 \%)$ & $>0.05$ \\
\hline superior migration & $9(13.2 \%)$ & $6(9.0 \%)$ & \\
\hline inferior migration & $20(29.4 \%)$ & $22(32.8 \%)$ & \\
\hline Pain duration(months) & $6.1 \pm 1.7$ & $5.7 \pm 2.3$ & \\
\hline Group A: the posterior longitudinal ligament was resected; Group B: the posterior longitudinal & & \\
\hline ligament was remaning. & & & \\
\hline
\end{tabular}

In the group A, Surgery was performed at the L3/4, L4/5, and L5/S1 levels in 6 (8.8\%), 51 (75\%), and 11 patients $(16.1 \%)$, respectively. The herniated disk types were central in 7 patients $(10.3 \%)$, paracentral in 32patients (47.1\%), superior migration in 9 patients (13.2\%) and inferior migration in 20 patients $(29.4 \%)$. In the group B, Surgery was performed at the L3/4, L4/5, and L5/S1 levels in 7 (10.4\%), 49 (73.1\%), and 11 patients $(16.4 \%)$, respectively. The herniated disk types were central in 5 patients $(7.5 \%)$, paracentral in 30 patients (44.8\%), superior migration in 6 patients (9.0\%) and inferior migration in 22 patients (32.8\%). 
The mean operation time of the group $A$ and the group $B$ respectively, were $82.7 \pm 18.5$ min and $115.6 \pm$ $24.6 \mathrm{~min}(\mathrm{P}<0.01)$. The average blood loss was $12.6 \pm 5.5 \mathrm{ml}$ and $14.2 \pm 7.3 \mathrm{ml}$ and there was no difference between the two groups. The postoperative VAS scores and ODI were significantly higher than those before surgery in two groups $(P<0.01)$. The differences in the results were not significant between the two groups $(P>0.05)$ (Table 2$)$.

Table 2

Summary of clinical outcomes

\begin{tabular}{|c|c|c|c|}
\hline Item & group A & group B & $P$ value \\
\hline Operation time(min) & $82.7 \pm 18.5$ & $115.6 \pm 24.6$ & $<0.01$ \\
\hline Blood loss (ml) & $12.6 \pm 5.5$ & $14.2 \pm 7.3$ & $>0.05$ \\
\hline Follow-up period (months) & 26.8 & 27.2 & $>0.05$ \\
\hline Preoperative VAS & $8.15 \pm 0.93$ & $8.23 \pm 0.87$ & $>0.05$ \\
\hline Last follow-up VAS & $2.30 \pm 1.5$ & $2.56 \pm 1.84$ & $>0.05$ \\
\hline Preoperative ODI (\%) & $63.1 \pm 17.2$ & $65.0 \pm 15.4$ & $>0.05$ \\
\hline Last follow-up ODI (\%) & $10.5 \pm 5.3$ & $11.6 \pm 4.6$ & $>0.05$ \\
\hline \multicolumn{4}{|l|}{ MacNab evaluation(n) } \\
\hline Excellence & 35 & 33 & \\
\hline Good & 27 & 28 & \\
\hline Fair & 4 & 5 & \\
\hline Poor & 2 & 1 & \\
\hline Excellence/good rate & $91.2 \%$ & $91.0 \%$ & $>0.05$ \\
\hline \multicolumn{4}{|l|}{ Complications(n) } \\
\hline Dural tear & 0 & 0 & \\
\hline Wound infection & 0 & 0 & \\
\hline Nerve root injury & 0 & 0 & \\
\hline Dysesthesia & 4 & 5 & \\
\hline Transient motor weakness & 2 & 1 & \\
\hline Complication rate & $8.8 \%$ & $8.9 \%$ & $>0.05$ \\
\hline Recurrence(n,\%) & $1(1.4 \%)$ & $2(2.9 \%)$ & $>0.05$ \\
\hline
\end{tabular}


For the modified MacNab criteria, the clinical outcome at final follow-up was excellent in 35 patients, good in 27, and fair in 4, excellent/good ratio is $91.2 \%$ in group A and excellent in 33 patients, good in 28, and fair in 5 with an excellent/good ratio of $91.0 \%$ in group $B$. There was no significant difference in the excellent/good ratio between the two groups $(P>0.05)$. In the series of patients, No serious complications, such as dural tear, wound infection or persistent nerve root injury, were observed. There are 4 patients in group $A$ and 5 patients in group $B$ developed postoperative dysesthesia. 3 patients ( 2 in group A, 1 in group B) experienced transient motor weakness. All of whom recovered with conservative treatment from 4 to 12 weeks. There was no significant difference in the complication rate between the two groups $(P>0.05)$. 1 patient in group $A$ and 2 patients in group $B$ had a recurrent disc herniation 3-6 months after surgery. Although the recurrence rate is higher in group $B$, There was no significant difference between the two groups $(P>0.05)$. The flexion-extension $x$-ray indicated that all cases in none of the patients in either group had increased spinal instability after surgery.

\section{Discussion}

For decades, minimally invasive spine surgery has been increasingly popular in treating LDH for multiple advantages compared with the open technique: such as less invasive, decreased blood loss, faster function recovery, shorter length of hospitalization, and lower risk of spinal instability[9-11].PETD via inside-and-out approach has become one of the most common minimally invasive spinal surgeries since Yeung et al. developed the spinal endoscopic YESS system in 2002[7, 12].Many studies have indicated that this technique could achieve good results with effective nerve root decompression, low complication rates for most types of LDH[13-16]. Yeung et al[7] reported 307 cases of LDH treated with the this technique followed-up for more than one year, and the excellent -to -good rate was $89.3 \%$. Similarly, in a study of 219 LDH patients (167 paramedian and 52 central herniations), the clinical outcomes showed the excellent or good rate was $88.1 \%$ with PETD via inside-and-out approach.

In the surgical technique, the working channel is first to be inserted into the disc just underneath the herniated nucleus pulposus, then, the channel is gradually diverted into the spinal canal as decompression progresses. During this procedure, if the posterior longitudinal ligament is found ruptured, the resection is recommended[17]. However, if the posterior longitudinal ligament is intact, whether the posterior longitudinal ligament is needed resection is controversial significantly[8].

Theoretically, retaining the posterior longitudinal ligament is beneficial to maintain spinal stability and prevent the reherniation of nucleus pulposus; On the other hand, it might increase the risk of free nucleus pulposus tissues left in spinal canal leading to inadequate decompression.

To our knowledge, no study has assessed the comparative curative effect between the two different surgical methods. In the current study, the results showed both methods had the same satisfactory clinical efficacy with similar complication rate. For the patients in group A, although the posterior longitudinal ligament is not resected, the careful detection underneath the herniated nucleus pulposus and in the spinal canal was made to confirm no residual nucleus pulposus tissues, which guarantee the 
adequate decompression as in group $B$. The recurrence rate is higher in group $B$, however, there was no significant difference between the two groups. The flexion-extension x-ray indicated that all cases in none of the patients in either group had increased spinal instability after surgery. The reason we think is that the posterior longitudinal ligament is only one aspect of maintaining spinal stability, the supraspinous ligament, interspinous ligament, capsule of facet joint was intact in both methods which play an important role in maintaining the spinal stability. The main difference between the two methods is patients in group B experienced longer operation time which may decrease patients' satisfaction under intraoperative local anesthesia. So, in our opinion, the resection of the posterior longitudinal ligament is unnecessary when the ligament is intact.

The retrospective nature of this study is a limitation. Studies of multicenter, randomized controlled trials should be conducted to compare the clinical efficacy of the two different surgical methods of LDH treated by PETD via inside-and-out approach.

\section{Conclusions}

Whether the posterior longitudinal ligament is resected do not make any difference on curative effect in PETD via inside-and-out approach, and remaining the ligament when it's intact is preferred because of its shorter operation time.

\section{Abbreviations}

LDH: Lumbar disc herniation;PELD:percutaneous endoscopic lumbar discectomy; PETD:percutaneous endoscopic transforaminal discectomy; PEID:percutaneous endoscopic interlaminar discectomy; VAS:Visual Analogue Score; ODI:Oswestry Disability Index.

\section{Declarations}

\section{Availability of data and materials}

The spinal internal fixation devices including pedicle screws, rod, and cage in surgical procedure are available and produced by Weigao Orthopedic, Shandong,China. The patients' data were collected in Chinese PLA General Hospital, the First Medical Center.

\section{Ethics approval and consent to participate}

This study was conducted with approval from the Ethics Committee of Chinese PLA General Hospital and was performed in accordance with the Declaration of Helsinki. Written informed consent was obtained from all participants. 


\section{Consent for publication}

Not applicable.

\section{Competing interests}

The authors declare that they have no competing interests.

\section{Funding}

This study was funded by the National Natural Science Foundation of China (Grant No. 8197090753), the Application of Clinical Features of Capital City of Science and Technology Commission China BEIJING Special subject (Z181100001718180), and Medical big data and artificial intelligence of PLA General Hospital research project (2019MBD-022).

\section{Authors' contributions}

All authors have read and approved the final manuscript. WH. H was involved in the study design, data collection, drafting and revising of the manuscript. FQ.H and CH. L were involved in the study design, analysis and interpretation of the data, drafting and revising of the manuscript.WB. $L$ was involved in the data collection. J.Y and J.L were involved in the analysis and interpretation of data. Y.W. was involved in the study design, data collection, analysis and interpretation of the data. XS. Z, L.L and T.L were involved in the study design, drafting and revising of the manuscript and has given final approval.

\section{Acknowledgements}

Not applicable.

\section{References}

1. Du J, Tang X, Jing X, Li N, Wang Y, Zhang X. Outcomes of percutaneous endoscopic lumbar discectomy via a translaminar approach, especially for soft, highly down-migrated lumbar disc herniation. International orthopaedics. 2016;40(6):1247-52.

2. Jensen TS, Albert HB, Soerensen JS, Manniche C, Leboeuf-Yde C. Natural course of disc morphology in patients with sciatica: an MRI study using a standardized qualitative classification system. Spine. 2006;31(14):1605-12. discussion 1613.

3. Kim HS, Yudoyono F, Paudel B, Kim KJ, Jang JS, Choi JH, Chung SK, Kim JH, Jang IT, Oh SH, et al. Analysis of Clinical Results of Three Different Routes of Percutaneous Endoscopic Transforaminal Lumbar Discectomy for Lumbar Herniated Disk. World neurosurgery. 2017;103:442-8. 
4. Nakamae T, Fujimoto Y, Yamada K, Nakanishi K, Kamei N, Yoshizaki K, Adachi N. Transforaminal percutaneous endoscopic discectomy for lumbar disc herniation in athletes under the local anesthesia. Journal of orthopaedic science: official journal of the Japanese Orthopaedic Association. 2019;24(6):1015-9.

5. Kambin P, Zhou L. Arthroscopic discectomy of the lumbar spine. Clinical orthopaedics and related research 1997(337):49-57.

6. Yeung AT. The evolution of percutaneous spinal endoscopy and discectomy: state of the art. Mt Sinai J Med. 2000;67(4):327-32.

7. Yeung AT, Tsou PM. Posterolateral endoscopic excision for lumbar disc herniation: Surgical technique, outcome, and complications in 307 consecutive cases. Spine. 2002;27(7):722-31.

8. Wang Y, Zhang W, Lian L, Xu J, Ding W. Transforaminal Endoscopic Discectomy for Treatment of Central Disc Herniation: Surgical Techniques and Clinical Outcome. Pain Physician. 2018;21(2):E113-23.

9. Anichini G, Landi A, Caporlingua F, Beer-Furlan A, Brogna C, Delfini R, Passacantilli E. Lumbar Endoscopic Microdiscectomy: Where Are We Now? An Updated Literature Review Focused on Clinical Outcome, Complications, and Rate of Recurrence. BioMed research international. 2015;2015:417801.

10. Gotecha S, Ranade D, Patil SV, Chugh A, Kotecha M, Sharma S, Punia P. The role of transforaminal percutaneous endoscopic discectomy in lumbar disc herniations. Journal of craniovertebral junction spine. 2016;7(4):217-23.

11. Choi KC, Kim JS, Lee DC, Park CK. Percutaneous endoscopic lumbar discectomy: minimally invasive technique for multiple episodes of lumbar disc herniation. BMC Musculoskelet Disord. 2017;18(1):329.

12. Song H, Hu W, Liu Z, Hao Y, Zhang X. Percutaneous endoscopic interlaminar discectomy of L5-S1 disc herniation: a comparison between intermittent endoscopy technique and full endoscopy technique. J Orthop Surg Res. 2017;12(1):162.

13. Yeung AT, Yeung CA. Minimally invasive techniques for the management of lumbar disc herniation. The Orthopedic clinics of North America. 2007;38(3):363-72. abstract vi.

14. Gore S, Yeung A. The "inside out" transforaminal technique to treat lumbar spinal pain in an awake and aware patient under local anesthesia: results and a review of the literature. International journal of spine surgery $2014,8$.

15. Morgenstern R, Morgenstern C, Yeung AT. The learning curve in foraminal endoscopic discectomy: experience needed to achieve a 90\% success rate. SAS journal. 2007;1(3):100-7.

16. Choi G, Kang HY, Modi HN, Prada N, Nicolau RJ, Joh JY, Pan WJ, Lee SH. Risk of developing seizure after percutaneous endoscopic lumbar discectomy. J Spin Disord Tech. 2011;24(2):83-92.

17. Yoshinari H, Tezuka F, Yamashita K, Manabe H, Hayashi F, Ishihama Y, Sugiura K, Takata Y, Sakai T, Maeda T, et al: Transforaminal full-endoscopic lumbar discectomy under local anesthesia in awake and aware conditions: the inside-out and outside-in techniques. Current reviews in musculoskeletal medicine 2019:311-317. 


\section{Figures}
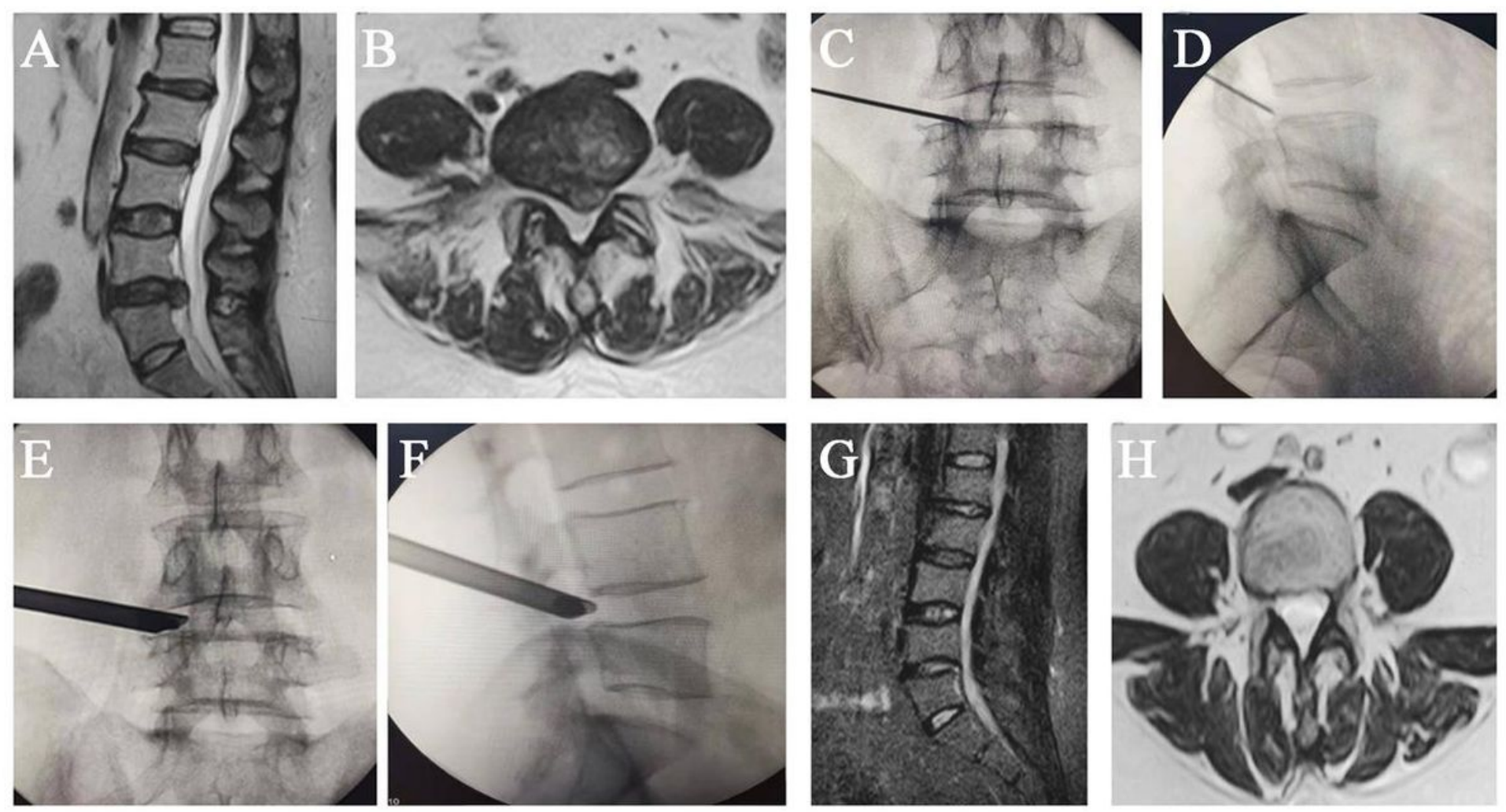

\section{Figure 1}

Percutaneous endoscopic transforaminal discectomy for a 50-year-old female patient. A,B Preoperative MRI image showing L4/5 disc herniation disc and compression of left L5 nerve root. C. Intraoperative anteroposterior fluoroscopy confirming the needle positioned on the medial pedicle edge. D. Lateral fluoroscopy confirming that the needle positioned on the posterior edge of the vertebral body. E, F. The working cannula was positioned at the appropriate place. G,H. 12 month postoperative MRI showing adequate decompression of nerve root and dura. 


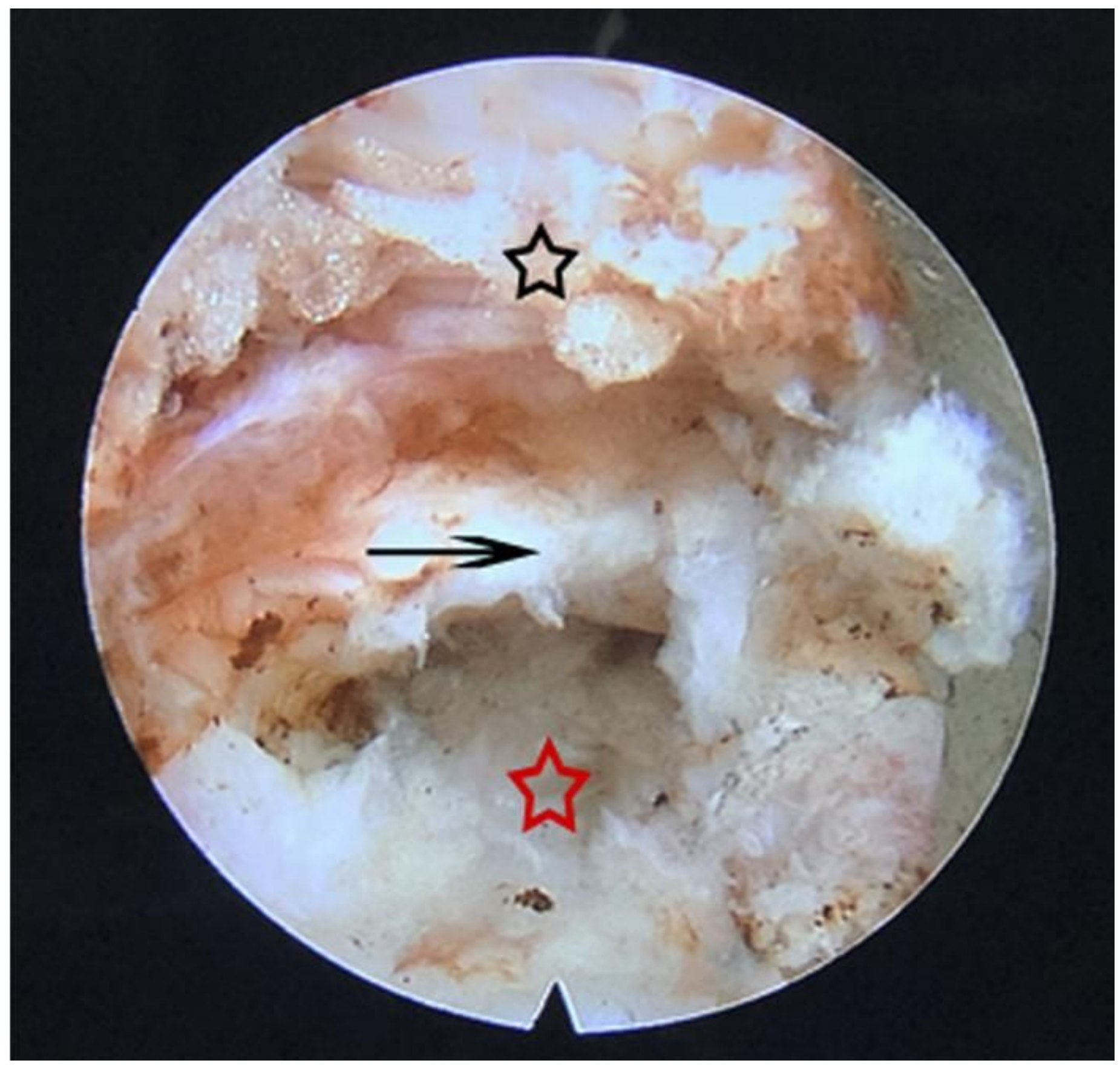

\section{Figure 2}

Intraoperative view. The posterior longitudinal ligament was in the middle (arrow), the top part is spinal canal (black star) and the bottom part is herniation (red star). 